\title{
An energy landscape model for glass-forming liquids in three dimensions
}

\author{
Ulf R. Pedersen, Tina Hecksher, Jeppe C. Dyre, Thomas B. Schrøder* \\ Department of Mathematics and Physics (IMFUFA), DNRF centre 'Glass and Time', Roskilde University, \\ Postbox 260, DK-4000 Roskilde, Denmark
}

Available online 8 September 2006

\begin{abstract}
We present a three-dimensional lattice-gas model with trivial thermodynamics, but non-trivial dynamics. The model is characterized by each particle having its own random energy landscape. The equilibrium dynamics of the model were investigated by continuous time Monte Carlo simulations at two different densities at several temperatures. At high densities and low temperatures the model captures the important characteristics of viscous liquid dynamics. We thus observe non-exponential relaxation in the self part of the density auto-correlation function, and fragility plots of the self-diffusion constant and relaxation times show non-Arrhenius behavior.

(C) 2006 Elsevier B.V. All rights reserved.
\end{abstract}

PACS: 64.70.Pf

Keywords: Diffusion and transport; Glass transition; Monte Carlo simulations; Structural relaxation

\section{Introduction}

A mechanical system of $N$ spherically symmetric particles is completely characterized by its so-called energy landscape, the graph of the potential energy function $U\left(\mathbf{r}_{1}, \ldots, \mathbf{r}_{N}\right)$ in $3 N+1$ dimensions. As suggested by Goldstein in his pioneering 1969 paper [1], the energy landscape is particularly useful for elucidating the dynamics of highly viscous liquids. This is because viscous liquid dynamics are dominated by jumps over barriers much larger than $k_{\mathrm{B}} T$; most time is spent on vibrations around local energy minima of the landscape. However, it was only after the work of Stillinger and Weber in the 1980s [2,3] and the enormous growth in use of computer simulations in the 1990s that the energy landscape became a dominant paradigm in the study of viscous liquids [4-11]. For recent reviews see, e.g., $[12,13]$.

It is difficult to imagine a complex high-dimensional landscape, but an obvious idea is to assume that there is

\footnotetext{
${ }^{*}$ Corresponding author.

E-mail address: tbs@ruc.dk (T.B. Schrøder).
}

an element of randomness in the landscape. In this philosophy one follows Wolynes, who argued that some phenomena occurring in a specific complex system are typical of those that occur in most systems chosen randomly out of an ensemble of possible systems [14].

A possible disordered landscape consists of a highdimensional lattice with random, uncorrelated energies chosen, e.g., according to a Gaussian, with nearest-neighbor Metropolis dynamics. This model, which has trivial thermodynamics, has been shown to reproduce a number of observed properties of viscous liquids, and the low-temperature dynamics of the model are understood to be dominated by site percolation $[15,16]$. However, the model does not have a meaningful thermodynamic limit; if the distribution of energies is chosen such that the mean energy is extensive, the relaxation times are not intensive. The problem, of course, is that a single nearest neighbor jump on the lattice changes the energy by an extensive amount, effectively corresponding to a complete rearrangement of all molecules. Another problem is how dimensionality is reflected in the energy landscape. Many condensed matter systems behave differently in two, three and four 
dimensions. If this applies also for viscous liquids, it must somehow be reflected in the landscape.

The question we consider here is: Is it possible to construct a sensible 'generic' random landscape model? Such a model should obey the following requirements:

1. It should have a well-defined thermodynamic limit, i.e., extensive average energy and intensive relaxation times.

2. It should reflect the dimensionality of space.

3. All sites should be statistically equivalent, thereby ensuring translational invariance on the average.

\section{The model}

The energy landscape is attractive because it abstracts from three dimensions. Nevertheless, we would like to suggest that the simplest way to have a model obeying the requirements listed above is to return to three dimensions.

Consider a lattice gas in three dimensions. If random energies are assigned to the lattice sites, the system is described by Fermi statistics. This corresponds to particles in an external random potential, thus with no translational invariance and only the trivial self exclusion particle-particle interaction. A simple modification turns this model into a highly non-trivial model, namely to assume that each particle has its own energy landscape, i.e., the energy of the system is given by (where $\mathbf{r}_{i}$ is the lattice position of particle $i, \delta$ is the Dirac $\delta$-function, and $k$ is a positive constant):

$E=\sum_{i} \epsilon_{i}\left(\mathbf{r}_{i}\right)+k \sum_{i \neq j} \delta\left(\mathbf{r}_{i}-\mathbf{r}_{j}\right)$.

The first term is the energetic interaction; for each lattice site, $\mathbf{r}$, and each particle, $i$, the energy, $\epsilon_{i}(\mathbf{r})$, is chosen randomly from a probability distribution, $p(\epsilon) . \epsilon_{i}(\mathbf{r})$ and $\epsilon_{j}(\mathbf{r})$ are generally different random numbers if $i \neq j$, i.e., particles have different energy landscapes. The second term in Eq. (1) is the self exclusion particle-particle interaction; no more than one particle is allowed at each lattice site.

In this model all lattice sites are statistically equivalent. The model allows calculation of pressure and chemical potential, and has extensive thermodynamics and intensive relaxation times. In particular we expect that at high densities, there will be a jamming effect slowing down the dynamics considerably.

When comparing the simulation results of this model to results from molecular dynamics (MD) simulations, one should keep in mind that the model does not include the high frequency vibrations associated with 'cage-rattling'. The dynamics that are modeled here are the 'inherent dynamics' [11], i.e., the result of mapping the true dynamics onto a series of inherent structures (local minima in the $3 N-1$ dimensional energy landscape).

We make the simplest possible choice for the probability distribution $p(\epsilon)$ : the Box distribution $[p(\epsilon)=1,0 \leqslant \epsilon<1]$.
In this case the mean system energy per particle is easily found to be $\left(\beta \equiv 1 / k_{\mathrm{B}} T\right)$ :

$\frac{\langle E\rangle}{N}=\frac{1}{\beta}-\frac{1}{\exp (\beta)-1}$.

At low temperatures we thus get $\langle E\rangle=N k_{\mathrm{B}} T$.

\section{Simulation details}

The model was simulated on a three-dimensional $L \times L \times L$ cubic lattice using the $N$-fold way kinetic Monte Carlo method $[17,18]$ with continuous time. We use Metropolis transition rates with local Monte Carlo moves; if a particle jumping to a nearest-neighbor site brings the system from state $i$ to state $j$, the associated transition rate is given by

$\Gamma(i \rightarrow j)=\min \left[\Gamma_{0}, \Gamma_{0} \exp \left(-\beta\left(E_{j}-E_{i}\right)\right)\right]$.

Our length and time units are defined by setting the lattice unit $a \equiv 1$ and the fastest transition rate $\Gamma_{0} \equiv 1$.

Since each particle has its own energy landscape, the number of different site energies are given by $N \times L^{3}=$ $\rho \times L^{6}\left(\rho \equiv N / L^{3}\right)$. Storing these numbers in memory would put a severe constraint on how large systems we could simulate. Instead we utilize the 'ran4' random number generator [19] in the following way: each particle is assigned a 'particle-seed', and when needed this is used together with the appropriate site-index as input to 'ran4', which performs a series of bit operations to produce a uniform random deviate in the range $0.0-1.0$.

The simulations are carried out with two sets of periodic boundary conditions, one for each term in Eq. (1). We denote by $L$ the lattice length associated with the particle-particle interactions. The usage of 'ran4' makes it possible to use much larger energy landscapes: the lattice site index used as input to 'ran4' is a 32-bit integer, and for the energy landscapes we can therefore use a side-length of approximately 1000 (we use $L$ times an integer), i.e., for all practical purposes each particle has an energy landscape that is infinite.

As mentioned above, we impose locality on the Monte Carlo moves; particles can only jump to (vacant) nearestneighbor sites. Relaxing this requirement (letting a Monte Carlo move consist of a random particle interchange with a random particle/hole) gives us an efficient way to equilibrate the model: for all the state points investigated here the characteristic time for equilibration was found to be less than 5 time units. Equilibration runs were done for a time period of 1000 time units.

\section{Simulation results}

Two densities were simulated, each at a range of $\beta$-values, see Table 1. Our simulations agree with the analytical expression for the mean energy (Eq. (2)). Reported results are averages over eight independent simulations (eight 
Table 1

Parameters used in simulations

\begin{tabular}{llll}
\hline$\rho$ & $\rho_{\mathrm{h}} \equiv 1-\rho$ & $\beta$-Values & $L$ \\
\hline 0.992 & $8 \times 10^{-3}$ & $0,2,4, \ldots, 16$ & 10 \\
0.999 & $1 \times 10^{-3}$ & $0,1,2, \ldots, 13$ & 20
\end{tabular}

$\rho \equiv N / L^{3}, \rho_{\mathrm{h}}$ is the density of holes (unoccupied sites). $\beta \equiv 1 / k_{\mathrm{B}} T$.

different energy landscapes), and error-bars are estimated from fluctuations between these eight simulations.

\subsection{Mean-square displacement}

Fig. 1 shows the mean-square displacement, $\left\langle r^{2}(t)\right\rangle$, in a $\log -\log$ plot. These results look similar to what is found in MD simulations of viscous liquids (see e.g., [20]): At long times the dynamics are diffusive $\left(\left\langle r^{2}(t)\right\rangle \propto t\right)$, and this diffusive regime is preceded by a plateau that develops as the system is cooled. In MD simulations this developing
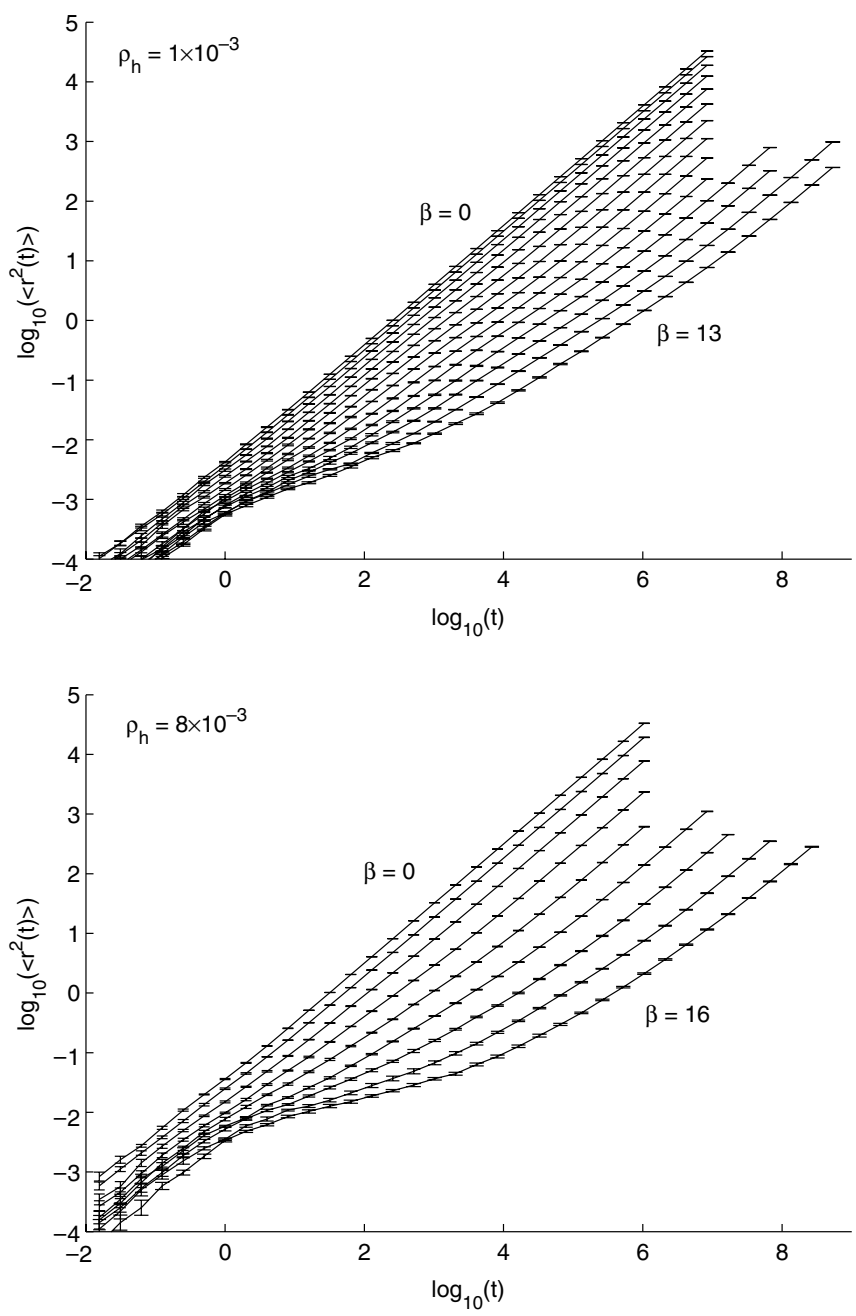

Fig. 1. Mean-square displacement, $\left\langle r^{2}(t)\right\rangle$, for $\rho_{\mathrm{h}}=1 \times 10^{-3}$ (upper panel) and $\rho_{\mathrm{h}}=8 \times 10^{-3}$ (lower panel). See Table 1 for further details. Data points are connected by straight lines. Error-bars indicate $95 \%$ confidence interval in $\left\langle r^{2}(t)\right\rangle$ estimated from fluctuations between eight independent samples (uncertainties only discernible at short times). plateau is attributed to 'cage rattling': particles vibrating in a cage consisting of the nearest neighbors. In this regime particles move considerably less than the inter-particle distance. This 'MD scenario' is obviously not what happens in this model; as discussed above the vibrations on length scales shorter than the inter-particle distance are not included in the model. Here the developing of a plateau means that after a particle has jumped to a nearest-neighbor site, the probability for jumping back to where it came from is (on average) larger than the probability for jumping to a new lattice site. This leads to a slowing down of the dynamics compared to diffusion dynamics, as allways seen in disordered systems [31]. Only when particles have
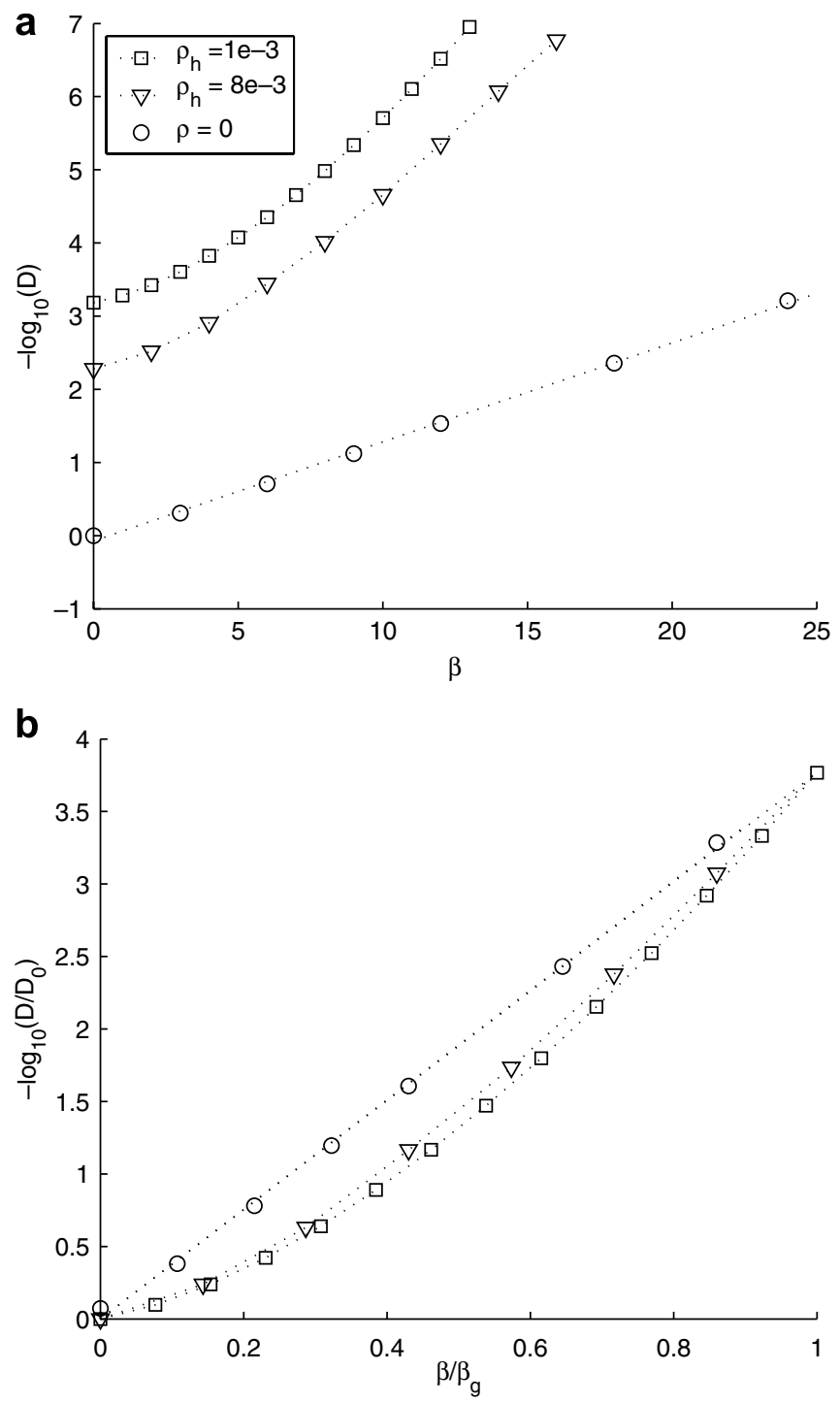

Fig. 2. Diffusion coefficients extracted from the mean square displacements in Fig. 1. For reference the diffusion coefficient in the $\rho=0$ limit (non-interacting particles) is included. Lower panel: same data as upper panel. $Y$-axis scaled by $D_{0} \equiv D(\beta=0)$. $X$-axis scaled by $\beta_{\mathrm{g}}$ to make the data collapse at $\beta / \beta_{\mathrm{g}}=1$. At the density $\rho_{\mathrm{h}}=1 \times 10^{-3}$ we define $\beta_{\mathrm{g}}=13$. For $\rho_{\mathrm{h}}=8 \times 10^{-3}$ and $\rho_{\mathrm{h}}=1$ empirical scaling was used to find $\beta_{\mathrm{g}}=13.95$ and $\beta_{\mathrm{g}}=30.9$, respectively. For $\rho=0$ a straight line was fitted to the data. For the high densities data points are connected with straight lines. 
jumped several times is this correlation between jumps lost whereupon the dynamics become diffusive. At short times $(t<1)$ a regime with $\left\langle r^{2}(t)\right\rangle \propto t$ is also seen - this is simply a consequence of the time scale being so short that particles never jump more than once.

In Fig. 2(a) we report the diffusion coefficients extracted from Fig. 1. For reference we show here also results for the $\rho=0$ limit, i.e., simulations with non-interacting particles (in this limit the model is obviously not a good model of a liquid). In the $\rho=0$ limit we find Arrhenius behavior $\left[D=D_{0} \exp (-\beta \Delta E)\right]$, as expected from percolation arguments [21]. In contrast, the higher densities show distinctive non-Arrhenius behavior; the model exhibits 'fragile' behavior. To facilitate comparison with Angell's fragility plot [22], we show in Fig. 2(b) the diffusion coefficients scaled in the following way: the $y$-axis is scaled with the diffusion coefficient at infinite temperature, $D_{0} \equiv D(\beta=0)$ (which scales with $\rho_{\mathrm{h}}$ [23]), and the $x$-axis is scaled with an 'inverse glass temperature', $\beta_{\mathrm{g}}$, which is here defined by the scaled diffusion coefficients being identical at $\beta / \beta_{\mathrm{g}}=1$ (and $\beta_{\mathrm{g}} \equiv 13$ for $\left.\rho_{\mathrm{h}}=1 \times 10^{-3}\right)$. The degree of fragility is observed to increase with increasing density (decreasing $\rho_{\mathrm{h}}$ ).

Fig. 3 shows the apparent activation energy, obtained by regarding $\Delta E$ in the Arrhenius expression as being temperature dependent:

$$
\Delta E=-\beta^{-1} \log \left(\frac{D}{D_{0}}\right) .
$$

In the $\rho=0$ limit $\Delta E$ as expected approaches the theoretical value, $E_{\mathrm{c}}=0.31$ [24]. At high densities, $\Delta E$ keeps increasing above this value, reflecting the non-Arrhenius behavior. There is an indication (particularly for $\rho_{\mathrm{h}}=$ $8 \times 10^{-3}$ ) that $\Delta E$ level off to a constant, indicating that there might be a crossover from non-Arrhenius to Arrhenius behavior, as seen e.g., in simulations of viscous silica [25]. The observed indication of a crossover to Arrhenius

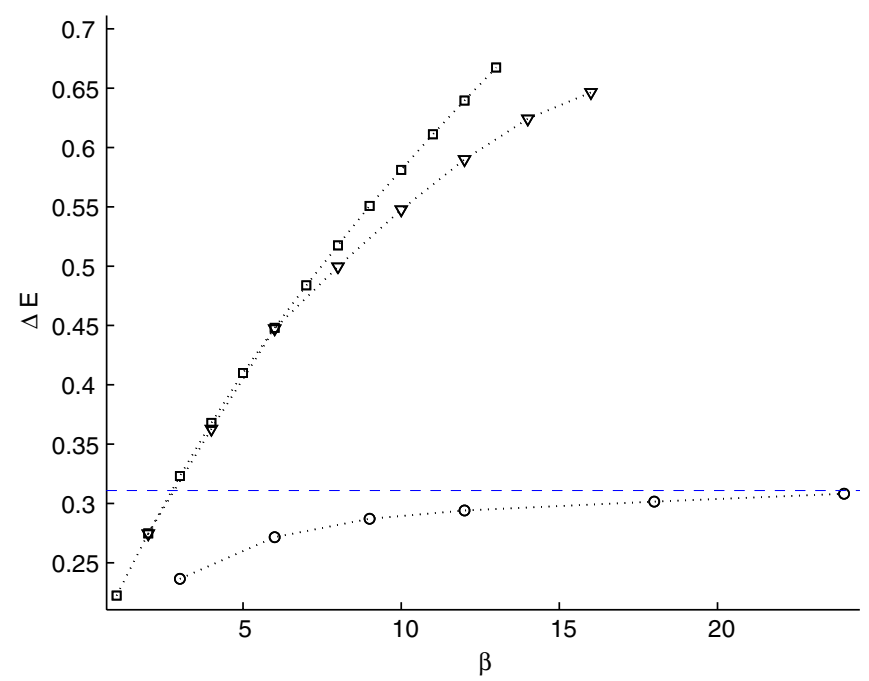

Fig. 3. Apparent activation energies calculated from Eq. (4). Squares: $\rho_{\mathrm{h}}=1 \times 10^{-3}$. Triangles: $\rho_{\mathrm{h}}=8 \times 10^{-3}$. Circles: $\rho_{\mathrm{h}} \rightarrow 1$ (i.e., $\rho=0$ limit, non-interacting particles). Data points are connected with straight lines. behavior might be related to 'hitting the bottom' of the energy landscape, but this point deserves further investigations.
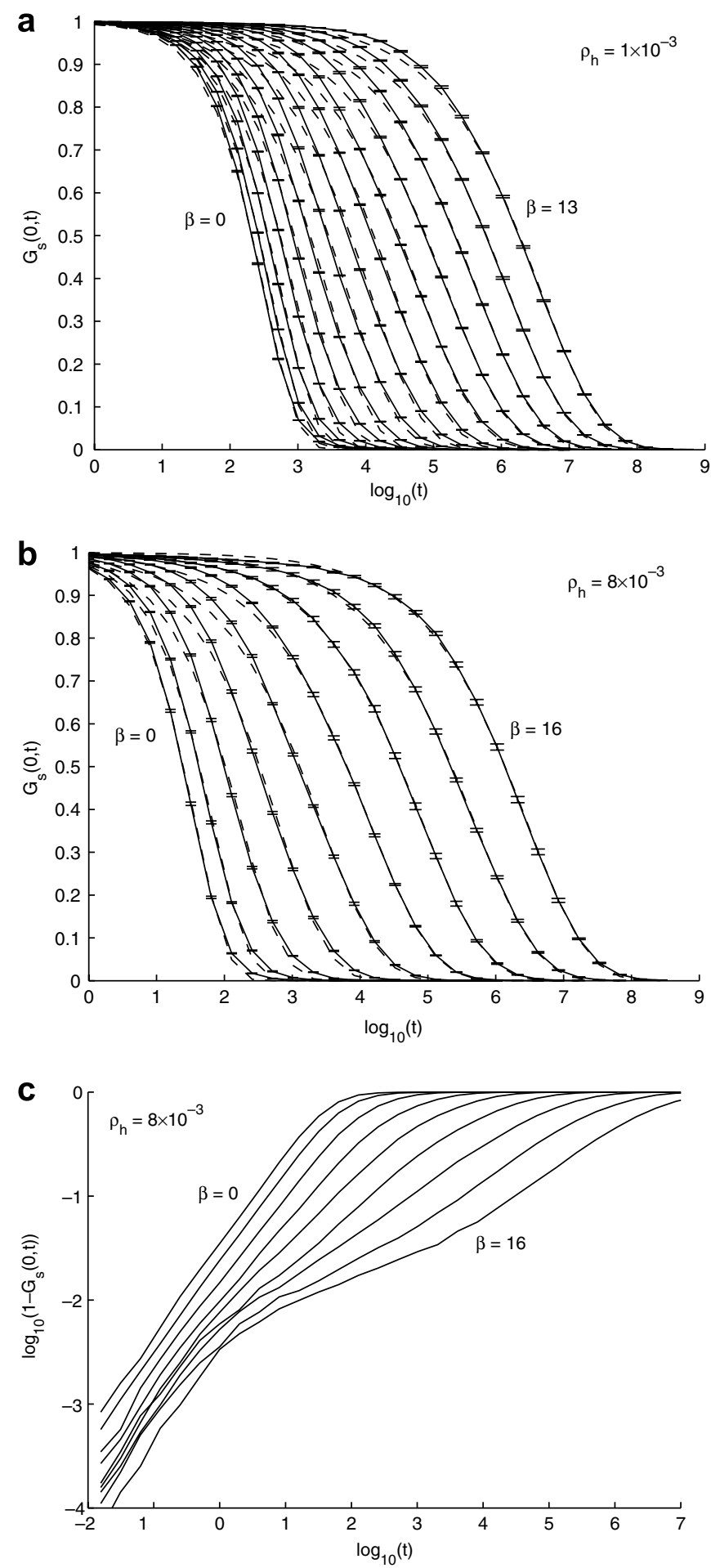

Fig. 4. Upper and middle panel: self part of the density-density correlation, $G_{\mathrm{s}}(0, t)$ for $\rho_{\mathrm{h}}=1 \times 10^{-3}$ and $\rho_{\mathrm{h}}=8 \times 10^{-3}$, respectively. Full lines are straight lines connecting data points. Error-bars indicate $95 \%$ confidence interval in $G_{\mathrm{s}}(0, t)$ estimated from fluctuations between eight independent samples. Dashed lines are fits to stretched exponentials, $\exp \left(-(t / \tau)^{\gamma}\right)$. Fits were done for $G_{\mathrm{s}}(0, t)<0.8$. Lower panel: $1-G_{\mathrm{s}}(0, t)$ for $\rho_{\mathrm{h}}=8 \times 10^{-3}$. 


\subsection{Density-density correlation (self part)}

In Fig. 4 we show the self part of the density auto-correlation, $G_{\mathrm{s}}(0, t)[26]$, i.e., the probability that a particle at time $t$ is at the same site as it was at time $t=0$. Full lines are straight lines connecting data points. Dashed lines are fits to stretched exponentials:

$f(t)=\exp \left(-(t / \tau)^{\gamma}\right)$.

The fits are not perfect, but they capture the main characteristics of the data. The fitting parameters are shown in Fig. 5. As for the diffusion constant (Fig. 2), the relaxation time $\tau$ exhibits non-Arrhenius behavior with an indication of a crossover to Arrhenius behavior at the lowest temperatures. The stretching exponent $\gamma$ decreases with $\beta$, indicating an increasing degree of non-exponential relaxation. Except for the lowest temperature at each density, the stretching exponent $\gamma$ seems to approach a constant close to 0.5 . A constant stretching exponent indicates time-temperature superposition (TTS), i.e., that the shape of the relaxation function is independent of temperature. We note
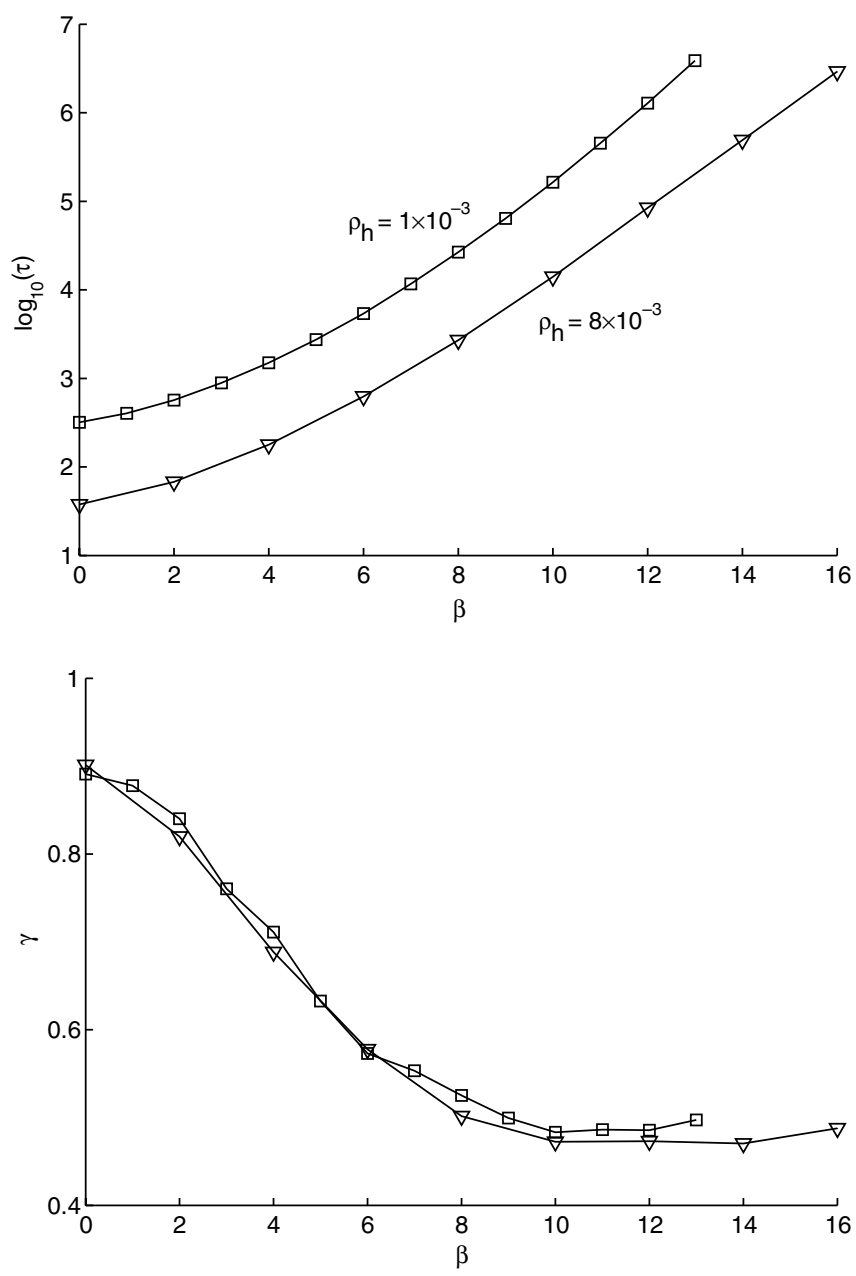

Fig. 5. Fitting parameters from fitting stretched exponentials to the return probability, $G_{\mathrm{s}}(0, t)$ (Fig. 4). Upper panel: relaxation time $\tau$. Lower panel: stretching exponent $\gamma$. Data points are connected by straight lines. that this behavior is consistent with experiments indicating that TTS is correlated to $\gamma=0.5$ [27]. Here we find at the very lowest temperatures an indication that the stretching exponent starts to increase again, which might be related to the apparent cross-over from non-Arrhenius to Arrhenius behavior discussed earlier. Simulations at lower temperatures are needed to investigate this question further.

Comparing Figs. 1-4(a) and (b), one might ask: 'why does a plateau develop in $\left\langle r^{2}(t)\right\rangle$ and not in $G_{\mathrm{s}}(0, t)$ ?'. The answer is, that there is indeed a plateau developing in $G_{\mathrm{s}}(0, t)$ - this can be seen in Fig. 4(c), where we have plotted $1-G_{\mathrm{s}}(0, t)$ (i.e., the fraction of particles that are contributing to $\left.\left\langle r^{2}(t)\right\rangle\right)$ in a log-log plot. In fact, at short times where no particle jumps more than once $\left\langle r^{2}(t)\right\rangle=1-G_{\mathrm{s}}(0, t)$. At the lowest temperature in Fig. 4(c) this relation holds to within $10 \%$ for $t \leqslant 100$.

We note that in the $\rho=0$ limit at low temperatures $G_{\mathrm{s}}(0, t)$ looks quantitatively different from what is seen in
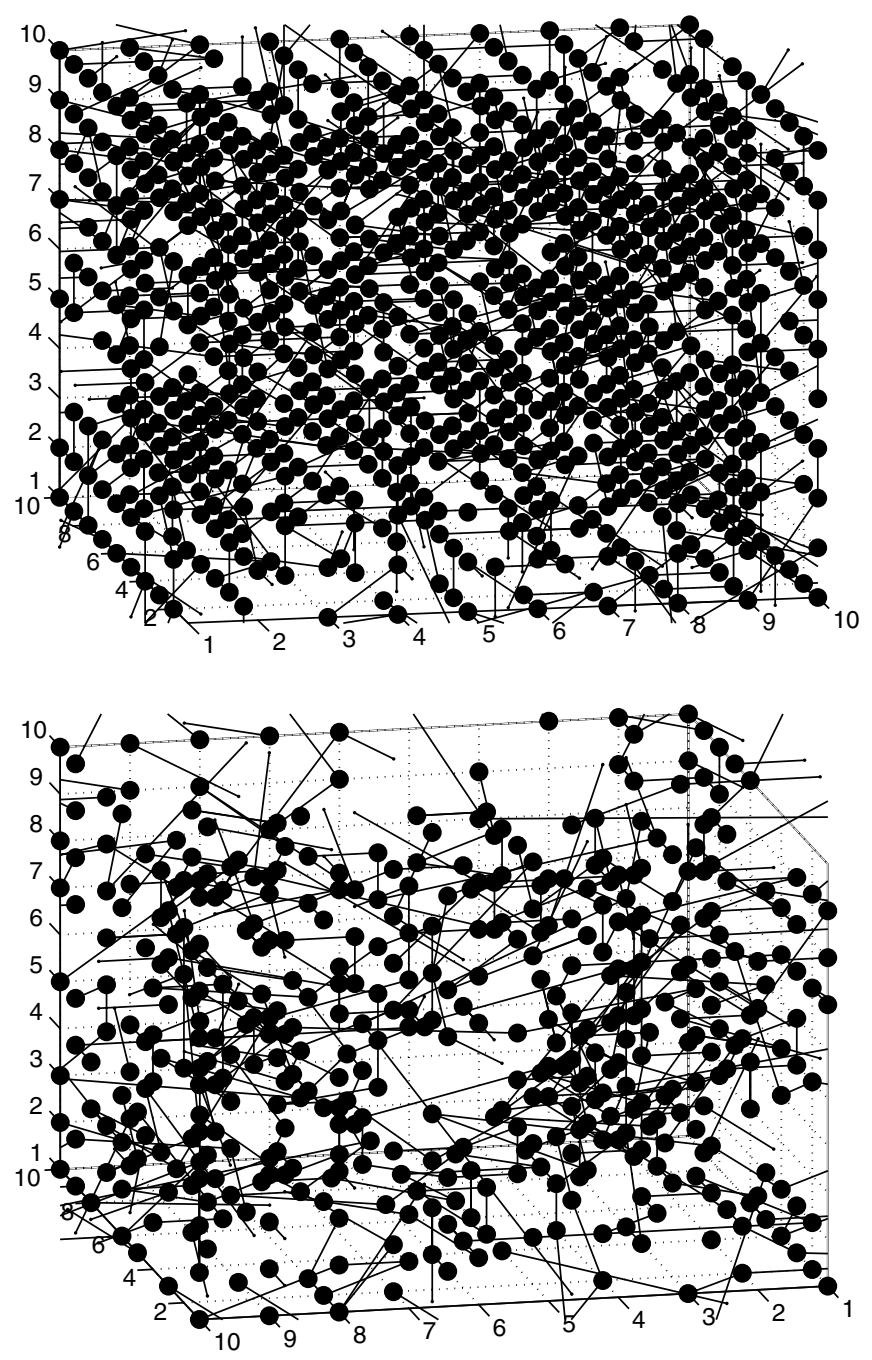

Fig. 6. Displacement vectors, at a time where $\left\langle r^{2}\right\rangle \approx 2$, for $\beta=0$ and 14 , respectively ( $\rho=0.992$, see Table 2 for details). The initial position of particles that moved to a new lattice site during the time interval is indicated by a filled circle, and the displacement vectors are shown as straight lines. 
Table 2

Parameters describing the two different sets of displacement vectors in Fig. 6

\begin{tabular}{llll}
\hline$\beta$ & $\left\langle r^{2}(t)\right\rangle$ & $t$ & $G_{\mathrm{s}}(0, t)$ \\
\hline 0 & 2.02 & $6.2 \times 10^{1}$ & $18.6 \%$ \\
14 & 2.06 & $2.0 \times 10^{5}$ & $54.1 \%$ \\
\hline
\end{tabular}

Note: Averages are here only over particles, not ensemble/time averages.

Fig. 4: there is a strong initial relaxation (related to jumps with $\Delta E<0)$, a stronger stretching $(\gamma \approx 0.3)$, and a final pronounced power law regime that starts at $G_{\mathrm{s}}(0, t) \approx 0.1$. This limit (which we again stress is not a good model for a liquid) is investigated in a separate paper [28].

\subsection{Dynamical heterogeneity}

It is well established that viscous liquids contain dynamical heterogeneities, i.e., if subsets of particles are defined by their dynamical properties, these tend to be correlated in time and/or space [29,30]. Fig. 6 indicates in a qualitative way that the model exhibits dynamical heterogeneity to an increasing degree as temperature is lowered. In Fig. 6 we show the displacement of particles at a time where $\left\langle r^{2}(t)\right\rangle \approx 2$ for $\beta=0$ and $\beta=14\left(\rho_{\mathrm{h}}=8 \times 10^{-3}\right)$. It is evident from the figure that the fraction of particles contributing to the mean square displacement (i.e., $\left.1-G_{\mathrm{s}}(0, t)\right)$ is smaller at the low temperature (see also Table 2), and that the positions of contributing particles are correlated in space.

\section{Conclusion}

A novel lattice-gas model of viscous liquids with extensive average energy and intensive relaxation times and diffusion coefficients has been proposed. The first results from simulations of the model have been presented. At high densities the model exhibits the two non's characterizing viscous liquids, non-exponential relaxation and non-Arrhenius temperature dependence of relaxation times and diffusion coefficients. The fragility increases with density. Finally, indications of a number of interesting features was found; (i) non-Arrhenius to Arrhenius transition, (ii) time-temperature superposition, (iii) dynamical heterogeneities.

\section{Acknowledgments}

This work was supported by a grant from the Danish National Research Foundation for funding the DNRF centre for viscous liquid dynamics 'Glass and Time'.

\section{References}

[1] M. Goldstein, J. Chem. Phys. 51 (1969) 3728.

[2] F.H. Stillinger, T.A. Weber, Phys. Rev. A 28 (1983) 2408.

[3] F.H. Stillinger, J. Chem. Phys. 88 (1988) 7818.

[4] R.J. Speedy, J. Chem. Phys. 100 (1994) 6684; R.J. Speedy, P.G. Debenedetti, Mol. Phys. 88 (1996) 1293; R.J. Speedy, Mol. Phys. 95 (1998) 169.

[5] C. Dasgupta, O.T. Valls, Phys. Rev. E 53 (1996) 2603, 59 (1999) 3123.

[6] F. Sciortino, P. Tartaglia, Phys. Rev. Lett. 78 (1997) 2385; F. Sciortino, W. Kob, P. Tartaglia, Phys. Rev. Lett. 83 (1999) 3214; A. Scala, F.W. Starr, E. La Nave, F. Sciortino, H.E. Stanley, Nature 406 (2000) 166.

[7] A. Heuer, Phys. Rev. Lett. 78 (1997) 4051; S. Büchner, A. Heuer, Phys. Rev. E 60 (1999) 6507; Phys. Rev. Lett. 84 (2000) 2168.

[8] S. Sastry, P.G. Debenedetti, F.H. Stillinger, Phys. Rev. E 56 (1997) 5533 ; Nature 393 (1998) 554.

[9] M. Schulz, Phys. Rev. B 57 (1998) 11319.

[10] L. Angelani, G. Parisi, G. Ruocco, G. Viliani, Phys. Rev. Lett. 81 (1998) 4648;

Phys. Rev. E 61 (2000) 1681.

[11] T.B. Schrøder, J.C. Dyre, J. Non-Cryst. Solids 235-237 (1998) 331; T.B. Schrøder, S. Sastry, J.C. Dyre, S.C. Glotzer, J. Chem. Phys. 112 (2000) 9834.

[12] D.J. Wales, Energy L, Scapes, Cambridge University, 2003.

[13] F. Sciortino, J. Stat. Mech. (2005) P05015.

[14] P.G. Wolynes, Acc. Chem. Res. 25 (1992) 513.

[15] H. Bässler, Phys. Rev. Lett. 58 (1987) 767.

[16] J.C. Dyre, Phys. Rev. B 51 (1995) 12276.

[17] A.B. Bortz, M.H. Kalos, J.L. Lebowitz, J. Comput. Phys. 17 (1975) 10.

[18] F.M. Bulnes, V.D. Pereyra, J.L. Riccardo, Phys. Rev. E 58 (1998) 86.

[19] W.H. Press, S.A. Teukolsky, W.T. Vetterling, B.P. Flannery, Numerical Recipes in Fortran 77, 2nd Ed., Cambridge University, 2003.

[20] W. Kob, H.C. Andersen, Phys. Rev. E 51 (1995) 4626.

[21] At low temperatures the dynamics are dominated by the largest energy a particle has to cross to go through the sample [16].

[22] C.A. Angell, in: K. Ngai, G.B. Wright (Eds.), Relaxations in Complex Systems, US Government Printing Office, Washington, DC, 1985 , p. 3.

[23] H. van Beijeren, R. Kutner, Phys. Rev. Lett. 55 (1985) 238.

[24] D. Stauffer, A. Aharony, Introduction to Percolation Theory, Revised 2nd Ed., Taylor \& Francis, 1994.

[25] J. Horbach, W. Kob, Phys. Rev. E 60 (1999) 3169.

[26] $G_{\mathrm{s}}(r, t)$ is the self part of the van Hove correlation function, i.e., the probability that particles are displaced the vector $\mathbf{r}$ in the time interval $t$.

[27] N.B. Olsen, T. Christensen, J.C. Dyre, Phys. Rev. Lett. 86 (2001) 1271.

[28] T.B. Schrøder, Numerical method for studying random walks on disordered structures, in preparation.

[29] H. Sillescu, J. Non-Cryst. Solids 243 (1999) 81.

[30] M.D. Ediger, Ann. Rev. Phys. Chem. 51 (2000) 99.

[31] J.C. Dyre, T.B. Schrøder, Rev. Mod. Phys. 72 (2000) 873. 